\title{
Desarrollo de un sistema matemático para la elaboración de tarifas volumétricas en especies arbóreas
}

\author{
Javier Jiménez Pérez ${ }^{1}$ \\ Oscar A. Aguirre Calderón ${ }^{1}$ \\ Eduardo J. Treviño Garza ${ }^{1}$ \\ Antonio Domínguez Calleros ${ }^{1}$
}

\section{RESUMEN}

En el manejo de ecosistemas forestales es una necesidad primordial, la elaboración de tablas y tarifas volumétricas apropiadas para especímenes arbóreos de mayor importancia económica, por lo que resulta ser una condición fundamental dentro de un ordenamiento ecológico sustentable, el establecimiento de estos sistemas dasométricos. El presente trabajo de investigación se basa en el desarrollo de un sistema matemático para la estimación volumétrica de especies arbóreas, mediante la aplicación de una asociación de modelos matemáticos. El resultado obtenido, después del análisis estadístico respectivo, muestra que el empleo de las tarifas volumétricas para evaluaciones globales dentro de los ecosistemas forestales representa una herramienta de gran utilidad, por lograr una alta precisión en la valoración de los recursos naturales renovables.

PALABRAS CLAVE:

Pinus pseudostrobus, volumen, tabla volumétrica, modelo matemático

\begin{abstract}
In forest ecosystems management it is a primary need to elaborate volume tables and tariffs appropriate for arboreal specimens with economic importance, therefore it turns out to be a fundamental condition in sustainable ecological planning, the establishment of these mensuration systems. The present study is based on the development of a mathematical system to estimate volumes of arboreal species, through the application of an association of mathematical models. The obtained result, after the respective statistical analysis, proves that the use of the volume tariffs for global evaluations within the forest ecosystems represents a tool of great usefulness, by achieving a high precision in considering how valuable the renewable natural resources are.
\end{abstract}

KEYWORDS:

Pinus pseudostrobus, volume, volume table, mathematical model.

1 Facultad de Ciencias Forestales. Universidad Autónoma de Nuevo León. Apdo. Postal 41. 6770 Linares, N.L. México. e-mail: jjimenez @ccr.dsi.uanl.mx.

Manuscrito recibido para su publicación el 8 de Octubre de 1998. 


\section{INTRODUCCION}

En los ecosistemas de clima templado, las especies del género Pinus y Quercus representan un alto significado ecológico y económico, por lo que el conocimiento de su dinámica resulta ser de gran importancia para el desarrollo y preservación de estas especies dentro de los ecosistemas forestales.

Las tablas de volumen ofrecen una medida alta de precisión en el cálculo volumétrico arbóreo, pero presentan un alto costo al tener una aplicación en la práctica. Tal controversia se tiene como premisa en este estudio, donde se plantea una nueva metodología denominada sistemas de estimación volumétrica 0 tarifas volumétricas, que representan un compromiso entre lo que es práctico y la precisión de las mediciones de recursos naturales.

\section{OBJETIVO}

El presente estudio tiene como principal objetivo el desarrollo de un nuevo procedimiento para la elaboración de tarifas volumétricas en especies de tipo arbóreo, a partir de modelos matemáticos para la determinación volumétrica de áreas arboladas utilizadas en el manejo forestal sostenible.

\section{ANTECEDENTES}

A inicios del siglo pasado se construyen las primeras tablas de volumen por Heinrich Cotta, el cual desarrolla la primera tabla de volumen para Fagus sylvatica, reconociendo que el volumen individual depende de la altura (Prodan, et al., 1997). La introducción de procesadores electrónicos facilitó en los últimos dos decenios la elaboración de tablas de volumen. Los países con una larga tradición forestal utilizan actualmente diferentes sistemas para determinar con una gran exactitud el volumen a nivel ecosistema forestal (Cao y Burkhart, 1980; Laasasenho, 1982; Sloboda, 1984; Martin, 1984; Reed y Byrne, 1985; McTague, 1992; Avery y Burkhart, 1994; Kramer y Akca, 1995; Philip, 1995; Laar y Acka, 1997; Prodan, et al., 1997).

Sloboda (1984) define sobre el efecto integral de la teoría de forma del árbol medio del rodal para el cálculo del volumen o coeficiente mórfico.

$$
v=\pi / 4 \int_{0}^{h}\left[\alpha_{1}(x / h)+\alpha_{2}(x / h) * d\right]^{2} d x
$$

Prodan et al. (1997) distinguen a las tarifas como funciones que estiman el volumen solo en función de una variable (diámetro). Ocasionalmente se encuentra una división en las diferentes clases de altura. La elaboración de tarifas volumétricas supone que la altura del árbol permanece como una constante o que el rango de medición se divide en clases de altura.

El método de construcción de tarifas se realiza a través de la aceptación de distintas variables asociadas al volumen (área basal, diámetro del árbol medio, etc.). Un ejemplo de esto son las tarifas volumétricas que proponen Turnbull et al. (1963) para los Estados Unidos de Norteamérica o Sosa (1976) para México.

Por la gran complejidad en la utilización de diferentes variables, se han construido una serie de tarifas de volumen, con el fin de disminuir los costos, a consecuencia de la precisión. En este sentido Kramer y Akca (1995) y Prodan et al. (1997) mencionan sobre las tarifas volumétricas para bosques de selección, la tarifa forma-altura, la tarifa de ALGAN, la 
tarifa de categorías de altura, así como la tarifa única.

Tarifa volumétrica del árbol medio: Krenn (1948) desarrolla tarifas para el cálculo del volumen de rodales, teniendo como fundamento matemático:

$$
\begin{aligned}
& V_{m}=f\left(D_{m}\right) \\
& H_{m}=f\left(D_{m}\right)
\end{aligned}
$$

$\mathrm{V}_{\mathrm{m}}=$ volumen del árbol medio del rodal

$D_{m}=$ diámetro del área basal media

$\mathrm{H}_{\mathrm{m}}=$ altura del área basal media

Prodan et al. (1997) subrayan las distintas ventajas sobre la tarifa volumétrica KRENN:

C Las mediciones en altura pueden ser remplazadas por estimaciones sencillas para el árbol medio.

C El cálculo del volumen del rodal es simple y requiere de poco tiempo.

C Los rodales permanecen con mucha probabilidad largo tiempo en la misma tarifa.

C Con mediciones periódicas se puede determinar fácilmente el incremento.

Kramer y Akca (1995) agregan ciertas desventajas del método KRENN:

C El error en volumen aumenta, cuando el cálculo del diámetro del área basal media $\left(D_{m}\right)$ y el número de individuos $\left(n_{i}\right)$ se obtienen de un muestreo representativo y existe la posibilidad de estimar el volumen en una tarifa diferente.

C Otros errores pueden surgir por la influencia de la relación $H_{m}$ y $D_{m}$ a consecuencia de un cambio por el aclareo.

Loetsch citado por Prodan et al. 1997) construye tarifas de volumen para los bosques de Indonesia, de acuerdo con la metodología propuesta por KRENN para varias especies arbóreas importantes (Pinus merkusii, Acacia decurrens, Antocephalus cadamba y Albizzia falcata).

$$
\mathrm{V}_{\mathrm{m}}=\mathrm{a}_{0}{ }^{*} \mathrm{D}_{\mathrm{m}}^{\mathrm{a} 1}
$$

$$
\log V_{m}=\log a_{0}+a_{1}{ }^{*} \log D_{m}
$$

Conn (1971) recomienda la utilización de regresiones polinomiales para la elaboración de tarifas del árbol medio en base a la siguiente fórmula:

$$
\begin{aligned}
V=f\left(D_{m}\right)= & a_{0}+a_{1}{ }^{*} D_{m}+a_{2}{ }^{*} D_{m^{2}} \\
& +\ldots+a_{n}{ }^{*} D_{m^{n}}
\end{aligned}
$$

Hoffmann (1982) propone una función para tarifas, la cual a partir del diámetro $\left(D_{m}\right)$ se estima el volumen medio de un árbol $\left(\mathrm{V}_{\mathrm{m}}\right)$.

$$
\begin{aligned}
& \mathrm{V}_{\mathrm{m}}=\operatorname{EXP}\left[\mathrm{a}_{0}+\mathrm{a}_{1}{ }^{*} \ln \left(\mathrm{D}_{\mathrm{m}}\right)+\mathrm{a}_{2}{ }^{*} \operatorname{In}^{2}\left(\mathrm{D}_{\mathrm{m}}\right)\right] \\
& \ln \left(\mathrm{V}_{\mathrm{m}}\right)=\ln ^{*} \mathrm{a}_{0}+\mathrm{a}_{1}{ }^{*} \ln \left(\mathrm{D}_{\mathrm{m}}\right)+\mathrm{a}_{2}{ }^{*} \ln ^{2}\left(\mathrm{D}_{\mathrm{m}}\right)
\end{aligned}
$$

Brister y Lauer (1985) presenta una tarifa media para Pinus taeda en los Estados Unidos de Norteamérica, la cual tiene como variables independientes la altura promedio y el diámetro medio del rodal.

$$
\mathrm{V}_{\mathrm{m}}=\mathrm{a}_{0}{ }^{*} \mathrm{D}_{\mathrm{m}}{ }^{\mathrm{a} 1}{ }^{*} \mathrm{H}_{\mathrm{m}}{ }^{\mathrm{a} 2}
$$

Tarifa forma-altura: Sobre la elaboración de tarifas de acuerdo con el principio de forma-altura, Kramer y Akco (1995) mencionan diferentes investigaciones, teniendo como base la variable formaaltura. La determinación del volumen 
mediante esta relación se fundamenta en la función:

$$
V=G^{*} F H
$$

$\mathrm{V}=$ Volumen del rodal

$\mathrm{G}=$ Area basal del rodal

$\mathrm{FH}=$ Coeficiente de forma-altura

El área basal del rodal se obtiene mediante muestreos representativos, por ejemplo, el muestreo de los seis árboles o el muestreo de dimensiones variables de BITTERLICH. La altura media de la especie se estima en el rodal y se utiliza como variable para determinar el valor en la tarifa forma-altura. La estimación del volumen mediante la tarifa forma-altura puede proveer información incorrecta, porque la altura media y la forma-altura del rodal se determinan con un alto grado de error (Kramer y Akco, 1995).

\section{METODOLOGIA}

Pinus pseudostrobus Lindl. El área de investigación se ubica en la Sierra Madre Oriental, donde se localizan cuatro tipos de vegetación: bosque de pino, bosque de pino-encino, bosque de encino y vegetación semiárida. Dentro de las formas de vegetación se distribuyen 12 especies diferentes de pino (Martínez, 1948). De éstas, pocas son las que presentan un potencial económicamente importante para el estado de Nuevo León.

Pinus pseudostrobus se localiza en México y América Central (Perry, 1991). En la Sierra Madre Oriental esta especie se desarrolla en una altitud de 800-2100 msnm. $P$. pseudostrobus manifiesta las siguientes características taxonómicas: hojas en grupos de 5, entre 17 a $24 \mathrm{~cm}$ de largo, color verde intenso. Conos ovoides o largamente ovoides, de color café claro.
Corteza lisa durante largo tiempo, en la madurez es áspera y agrietada. En su área natural de distribución alcanza una altura de 15 a $25 \mathrm{~m}$. Existe una semejanza taxonómica de esta especie con el grupo Montezumae (Martínez, 1948).

La importancia económica de esta especie radica en su amplia distribución en el norte y sur de México. Sus propiedades físicas y anatómicas han sido investigadas por diversos autores (entre ellos De la Paz y Olvera, 1981). La madera de Pinus pseudostrobus se usa principalmente para productos aserrados, durmientes para vías de ferrocarril y muebles.

Descripción general del área de estudio. El área de estudio se localiza en una fracción de la Sierra Madre Oriental, en los ejidos: La Trinidad, Pedro Carrizales, La Luz y La Purísima, enmarcados en los municipios de Montemorelos, Rayones e Iturbide en el estado de Nuevo León. Geográficamente converge esta región entre los 24/31' y 25/15' latitud norte y los 99/49' y 100/10' de longitud oeste. El área presenta una alta variabilidad altitudinal que oscila entre los 900 y 2200 m.

Método de investigación: En la elaboración de tablas volumétricas lo fundamental es analizar y definir los distintos tipos de estructuras de vegetación, para lograr un criterio en la elección de la muestra representativa en cada área. La decisión del número de individuos a cubicar en forma seccional para una descripción regional, así como su distribución por clases diamétricas es un conflicto que ha sido discutido ampliamente sin llegar a tener una conclusión correcta; sin embargo desde el punto de vista estadístico puede efectuarse cuando se tiene la información previa para calcular la variación existente en cada categoría diamétrica. El número de árbolesmuestra necesarios para la elaboración de una tabla volumétrica depende en gran medida de la variación existente en la forma 
estructural de los mismos. Cuanto más homogénea es la forma de la especie, menor será el número de observaciones requeridas (Jiménez 1988, 1990).

Pinus pseudostrobus exhibe dentro de su rango de amplitud en la Sierra Madre Oriental una gran heterogeneidad en cuanto a su forma. A causa de la variación en la forma de la especie, se eligió un número alto de muestras (563 individuos). La muestra se distribuyó teniendo como rango de amplitud diamétrica de $5 \mathrm{~cm}$ y de $5 \mathrm{~m}$ para categorías de altura. La variación con respecto al diámetro en Pinus pseudostrobus oscila entre $4.5 \mathrm{~cm}$ y 64.5 $\mathrm{cm}$ y en altura de $3.5 \mathrm{~m}$ hasta $27.4 \mathrm{~m}$ (Fig. 1).

Posteriormente se procedió a determinar estadísticamente la relación diámetro/altura. En este estudio se consideraron distintos modelos matemáticos, encontrando que la regresión de KORSUN (Kramer y Akca, 1995) presentan el mejor ajuste a la dispersión de los datos.

Función matemática de KORSUN

$$
h=\operatorname{EXP}\left[a_{0}+a_{1}{ }^{*} \ln (d)+a_{2}{ }^{*} \ln \left(d^{2}\right)\right]
$$

Función matemática para Pinus pseudostrobus:

$$
h=\operatorname{EXP}\left[\begin{array}{l}
0.4789628+227.8456 * \ln (d) \\
-113.5982 * \ln \left(d^{2}\right)
\end{array}\right]
$$

El coeficiente de correlación $(r)$ es de 0.9055 y el coeficiente de determinación $\left(r^{2}\right)$ de 0.8199 .

En la figura 2 se muestra la relación diámetro-altura que servirá para observar el desarrollo de la altura con un incremento diamétrico. La variación del modelo matemático en función de la dispersión auxiliará posteriormente en la elaboración de la tarifa media.

Para el desarrollo de modelos matemáticos en volumen y coeficiente mórfico se seleccionaron las variables dependientes: volumen con corteza (v) y coeficiente mórfico (f), y como variables independientes, el diámetro a $1.3 \mathrm{~m}$ (d) y la altura total (h) del árbol. Mediante transformaciones en las variables primarias independientes $(d, h)$ se establecieron las variables determinantes para analizar las distintas regresiones no lineales.

De la literatura se seleccionaron los criterios para elegir el mejor modelo matemático. Como criterios importantes fueron: tener un valor mínimo en el error estándar de la estimación (Sxy\%) y un máximo en el coeficiente de determinación $\left(r^{2}\right)$, asimismo contar con un número mínimo de variables independientes $(n)$ (Draper y Smith, 1980; Clutter et al., 1983).

\section{RESULTADOS}

Tabla volumétrica. En esta investigación se examinaron 55 diferentes modelos matemáticos volumétricos para Pinus pseudostrobus. Del número original de modelos de regresión, se redujo a 5 modelos sobre la base de los criterios estadísticos antes citados (Tabla 1). En el presente estudio se rechazó la utilización directa de las funciones de volumen por presentar en las categorías menores una gran variación en la predicción de valores.

La función matemática seleccionada (número 3), se fundamenta en el modelo biológico de volumen desarrollado por SLOBODA. Este modelo matemático se identifica con la dispersión de las observaciones, presentando un errorestándar mínimo y un número adecuado de variables independientes. 


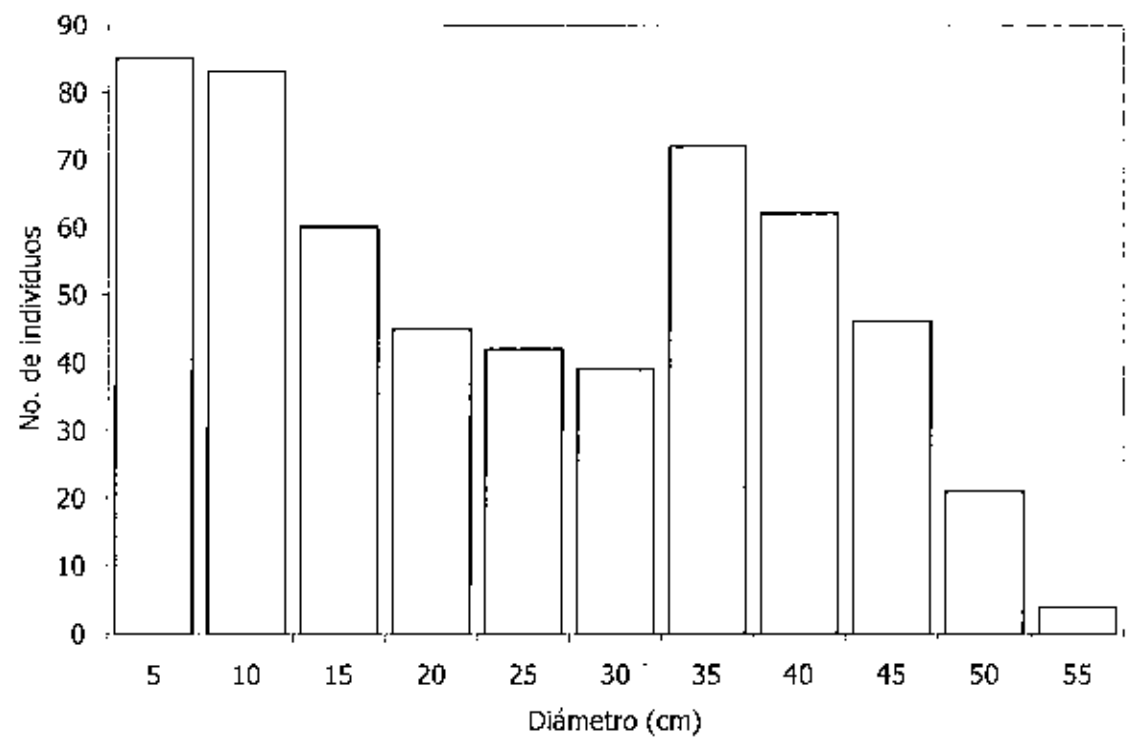

Figura 1. Distribución diamétrica de los individuos-muestra de Pinus pseudostrobus.

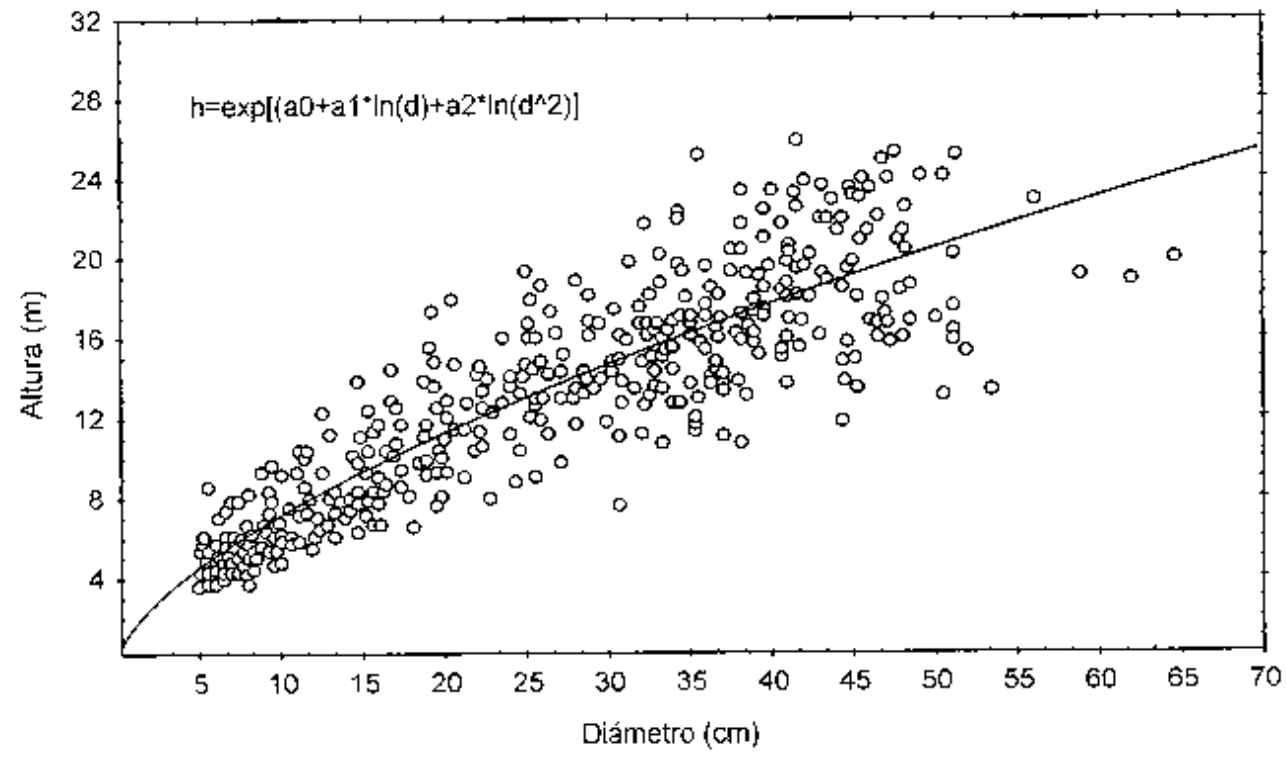

Figura 2. Relación diámétro-altura de acuerdo con el modelo matemático de KORSUN. 
Tabla 1. Modelos matemáticos en función del coeficiente mórfico (f).

\begin{tabular}{||c|l|c|c|c||}
\hline \hline NUMERO & \multicolumn{1}{|c|}{ MODELO MATEMATICO } & Sxy(\%) & $r^{2}$ & $n$ \\
\hline 1 & $f=\ln \left(d^{2}\right)+1 / d+1 / d^{2}+1 / d h+1 / d^{2} h$ & 9.61 & 0.8089 & 5 \\
\hline 2 & $f=\ln \left(d^{2}\right)+1 / d^{2}+1 / d$ & 9.76 & 0.7998 & 3 \\
\hline 3 & $f=1 / d+1 / h+1 / d^{2} h$ & 9.82 & 0.7984 & 3 \\
\hline 4 & $f=1 / d^{2}+1 / d$ & 10.50 & 0.7634 & 2 \\
\hline 5 & $f=1 / d^{2} h$ & 10.07 & 0.7859 & 1 \\
\hline
\end{tabular}

$$
V=a_{0}+a_{1}{ }^{*} h+a_{2}{ }^{*} d h+a_{3}{ }^{*} d^{2} h
$$

Debido a la amplia dispersión de los datos, y a la gran variabilidad existente entre los valores reales y predichos de Pinus pseudostrobus fue necesario efectuar una modificación sustancial al modelo matemático original. Esta transformación se basa en la utilización del coeficiente mórfico (f) como una variable de gran estabilidad. De la asociación anterior se obtuvo un error estándar porcentual de 9.8 y un coeficiente de determinación de $79.9 \%$.

$$
\begin{aligned}
f= & a_{0}+a_{1} *\left(1 / d^{2} h\right)+a_{2} *\left(1 / d^{2}\right)+a_{3} *(1 / d) \\
f= & 0.5071+1.6365 *\left(1 / d^{2} h\right)+8.9821^{*}\left(1 / d^{2}\right) \\
& +1.1432 *(1 / d)
\end{aligned}
$$

Para lograr una estimación correcta del volumen en base al modelo antes descrito, se elaboró una nueva función volumétrica, donde se anexa una constante absoluta $\left(a_{1}\right)$.
Tarifa volumétrica: Para desarrollar la tarifa volumétrica se determinó inicialmente la relación diámetro- altura en función del modelo matemático de KORSUN para cada una de las tarifas [superior (S), media (M) e inferior (I)]. El rango de variación de la altura se fraccionó en seis secciones de igual longitud relativa:

$$
h=\operatorname{EXP}\left[a_{0}+a_{1}{ }^{*} \ln (d)+a_{2}{ }^{*} \ln \left(d^{2}\right)\right]
$$

- Curva de altura superior (S)

$$
h=\operatorname{EXP}\left[\begin{array}{l}
0.700986+7.544241^{*} \ln (d) \\
-3.447358^{*} \ln \left(d^{2}\right)
\end{array}\right]
$$

- Curva de altura media (M)

$$
h=\operatorname{EXP}\left[\begin{array}{l}
0.4789628+227.8456 * \ln (d) \\
-113.5982^{*} \ln \left(d^{2}\right)
\end{array}\right]
$$

- Curva de altura inferior (I)

$$
h=\operatorname{EXP}\left[\begin{array}{l}
0.1911642+2.6900001^{*} \ln (d) \\
-1.020385 * \ln \left(d^{2}\right)
\end{array}\right]
$$

Para cada una de las funciones anteriores se definieron los coeficientes de regresión. Dichas funciones de 
diámetro/altura se utilizaron para determinar el volumen en cada tarifa volumétrica de acuerdo al modelo matemático de SLOBODA modificado. En la figura 3 se muestra la distribución de las tarifas volumétricas en función de la altura y diámetro.

En forma posterior, se determinó el rango de altura para la tarifa media, el cual tiene la finalidad de elegir la tarifa correcta en el diámetro $\left(d_{g}\right)$ y altura media del área basal $\left(h_{g}\right)$. Esta determinación esta en función al valor promedio obtenido en los muestreos representativos en los ecosistemas forestales. Cuando la altura media del área basal se encuentra dentro del rango de altura de la tarifa media (M), se acepta el volumen medio correspondiente. Se localiza la altura media del rodal arriba o abajo del rango de amplitud, entonces el volumen se obtiene de la tarifa superior o inferior.

En la Tabla 2 se muestra el ejemplo para Pinus pseudostrobus. El volumen se derivó para cada $0.2 \mathrm{~cm}$ y cada valor se estimó hasta cuatro dígitos.

En la Tabla 3 se observa la variación de la Tarifa Media con relación a la Tarifa Superior e Inferior. Este resultado muestra que la variación en las categorías diamétricas menores es mínima (8.8\%), pero a medida que las categorías son mayores, dicha variación se incrementa. A partir de la categoría diamétrica $20 \mathrm{~cm}$ dicha variación permanece constante (24.4\% $24.9 \%$ ).

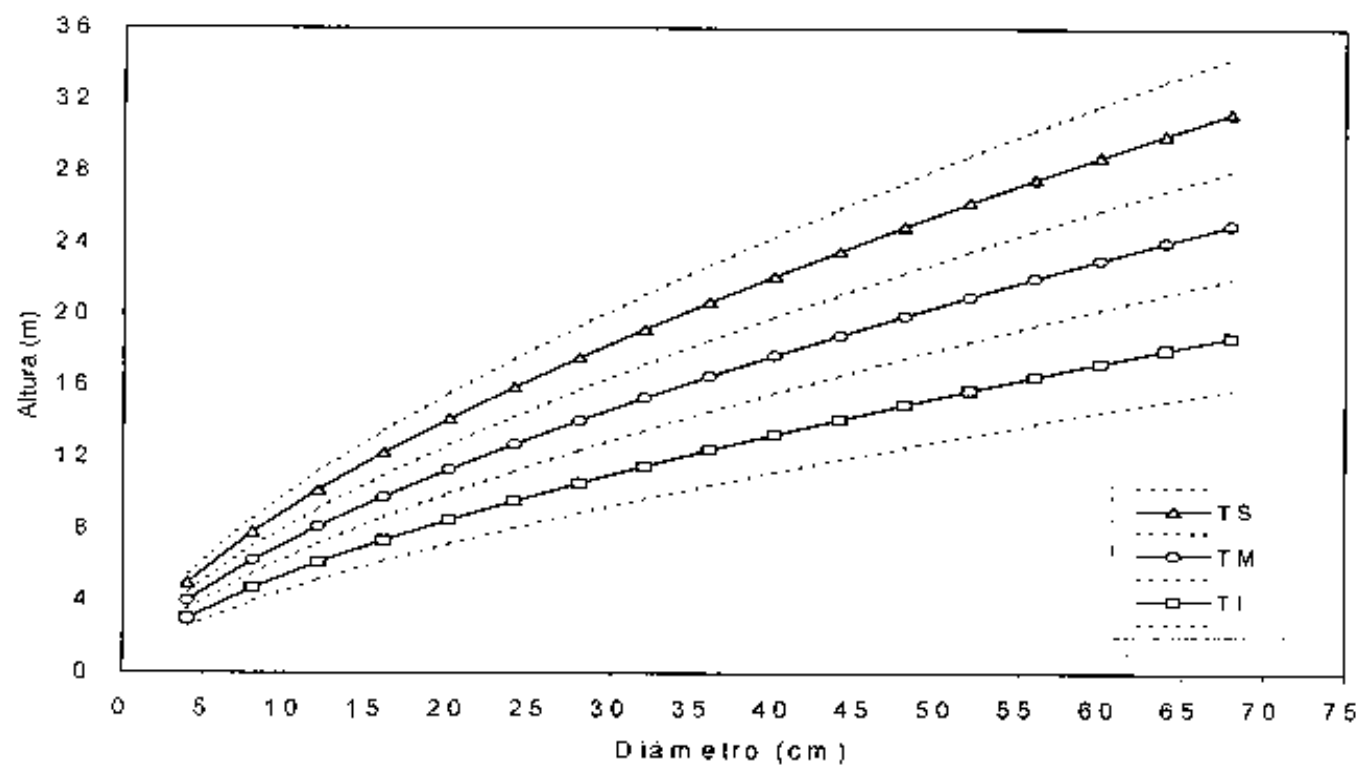

Figura 3. Distribución de las tarifas volumétricas en función del diámetro y altura. 
Tabla 2. Ejemplo tabular de la tarifa volumétrica en función del diámetro y altura media del área basal.

\begin{tabular}{|c|c|c|c|c|c|c|c|}
\hline $\begin{array}{l}\text { DIAMETRO }\left(\mathrm{d}_{\mathrm{g}}\right) \\
(\mathrm{cm})\end{array}$ & $\begin{array}{l}\text { TARIFA M } \\
\text { ALTURA }\left(\mathrm{h}_{\mathrm{g}}\right) \\
(\mathrm{m})\end{array}$ & TARIFA & 0.0 & 0.2 & 0.4 & 0.6 & 0.8 \\
\hline \multirow{3}{*}{5} & \multirow{3}{*}{$3.7-4.7$} & $S$ & 0.0074 & 0.0080 & 0.0086 & 0.0093 & 0.0101 \\
\hline & & $M$ & 0.0068 & 0.0072 & 0.0078 & 0.0083 & 0.0089 \\
\hline & & 1 & 0.0062 & 0.0065 & 0.0069 & 0.0073 & 0.0078 \\
\hline \multirow{3}{*}{6} & \multirow{3}{*}{$4.3-5.1$} & $S$ & 0.0108 & 0.0117 & 0.0216 & 0.0135 & 0.0145 \\
\hline & & M & 0.0095 & 0.0102 & 0.0109 & 0.0117 & 0.0125 \\
\hline & & 1 & 0.0082 & 0.0087 & 0.0093 & 0.0098 & 0.0104 \\
\hline \multirow{3}{*}{7} & \multirow{3}{*}{$4.8-5.9$} & $S$ & 0.0155 & 0.0166 & 0.0178 & 0.0190 & 0.0202 \\
\hline & & $M$ & 0.0133 & 0.0142 & 0.0151 & 0.0161 & 0.0171 \\
\hline & & 1 & 0.0111 & 0.0117 & 0.0124 & 0.0131 & 0.0139 \\
\hline \multirow{3}{*}{8} & \multirow{3}{*}{$5.1-6.6$} & $S$ & 0.0216 & 0.0229 & 0.0244 & 0.0259 & 0.0274 \\
\hline & & $M$ & 0.0181 & 0.0192 & 0.0204 & 0.0216 & 0.0228 \\
\hline & & 1 & 0.0147 & 0.0155 & 0.0164 & 0.0173 & 0.0182 \\
\hline \multirow{3}{*}{9} & \multirow{3}{*}{$5.6-7.2$} & $S$ & 0.0290 & 0.0307 & 0.0325 & 0.0343 & 0.0362 \\
\hline & & $M$ & 0.0241 & 0.0255 & 0.0269 & 0.0283 & 0.0298 \\
\hline & & 1 & 0.0192 & 0.0202 & 0.0212 & 0.0223 & 0.0234 \\
\hline \multirow{3}{*}{10} & \multirow{3}{*}{$6.1-7.7$} & $S$ & 0.0381 & 0.0401 & 0.0422 & 0.0444 & 0.0466 \\
\hline & & $\mathrm{M}$ & 0.0314 & 0.0330 & 0.0347 & 0.0364 & 0.0382 \\
\hline & & 1 & 0.0246 & 0.0258 & 0.0271 & 0.0284 & 0.0297 \\
\hline
\end{tabular}

Tabla 3. Variación volumétrica de las distintas categorías diamétricas.

\begin{tabular}{||c|c|c|c||}
\hline $\begin{array}{c}\text { DIAMETRO } \\
(\mathrm{cm})\end{array}$ & $\begin{array}{c}\text { VARIACION } \\
\text { VOLUMETRICA } \\
\text { PARA CADA TARIFA } \\
(\%)\end{array}$ & $\begin{array}{c}\text { DIAMETRO } \\
(\mathrm{cm})\end{array}$ & $\begin{array}{c}\text { VARIACION } \\
\text { VOLUMETRICA } \\
\text { PARA CADA TARIFA } \\
(\%)\end{array}$ \\
\hline 5 & 8.8 & 35 & 24.8 \\
\hline 10 & 21.3 & 40 & 24.9 \\
\hline 15 & 23.7 & 45 & 24.9 \\
\hline 20 & 24.4 & 50 & 24.9 \\
\hline 25 & 24.6 & 55 & 24.9 \\
\hline 30 & 24.8 & 60 & 24.9 \\
\hline
\end{tabular}




\section{DISCUSION Y CONCLUSION}

En el marco del manejo, inventario y monitoreo de ecosistemas forestales tiene un alto significado la precisión sobre la forma de estimar la variable volumen. La determinación volumétrica en la práctica forestal mexicana presenta una serie de errores, debido a la baja eficiencia de las tablas volumétricas. Con base en lo antes señalado, se desarrollaron nuevos sistemas de estimación volumétrica para Pinus pseudostrobus, especie importante en el noreste de México.

La distribución del volumen en las categorías diamétricas indica que la especie Pinus pseudostrobus presenta una gran variabilidad en su distribución y forma, por lo que su grado de error se acepta como adecuado. En comparación con la tabla volumétrica, la cual requiere de una gran precisión y un alto costo de medición, resulta la tarifa volumétrica con una diferencia no mayor a $9.76 \%$.

Es importante definir que las tarifas volumétricas resultan ser una opción más en la estimación volumétrica de ecosistemas forestales por ser una forma simple de valoración con una precisión adecuada y un costo mínimo en la evaluación. Teniendo como fundamento lo anteriormente señalado, la utilización de este nuevo sistema matemático ofrecerá la posibilidad de actuar bajo un principio práctico y preciso en la evaluación de los recursos forestales, principalmente en especies arbóreas económicamente importantes.

\section{REFERENCIAS}

Avery, T. y Burkhart. H. 1994. Forest Measurements. McGraw Hill, Inc. New York. 408 p.
Brister, G. y D. Lauer. 1985. A tariff system for loblolly pine. For. Sci. 31(1): 95-108

Cao, Q. y H. Burkhart. 1980. Cubic-foot volume of loblolly pine to any height limit. South. J. of Appl. For. 4: 166-168

Clutter, J., J.C. Fortson, L.V. Pienaar, G.H. Brister y R.L. Bailey. 1983. Timber management. a quantitative approach. John Wiley \& Sons. New York. 333 p.

Conn, G. 1971. Konstruktion eines Mittelstammtarifes. Dissertation. Freiburg. 101 p.

De la Paz P. O., C. y P. Olvera. C. 1981. Anatomía de la madera de 16 especies de coníferas. INIF. Bol. Téc. 69.

Draper, N. y H. Smith. 1980. Applied regression analysis. John Wiley and Sons. New York. 709 p.

Hoffmann, C. 1982. Die Berechnung von Tarifen für die Waldinventur Forstwiss. Cbl. 42: 24-36.

Jiménez, J. 1988. Aufstellung von Schaftholz-Massentafeln und Tarifen für Pinus pseudostrobus und Pinus teocote in einem Teil del Sierra Madre Oriental im Nordosten Mexikos. Disertation. Göttingen. 104 p.

Jiménez, J. 1990. Aplicación de un modelo matemático para elaborar tablas y tarifas de volumen. Un ejemplo con Pinus pseudostrobus. Reporte Científico No. 16. Facultad de Ciencias Forestales. Linares, Nuevo León. 48 p.

Kramer, H. y A. Akca. 1995. Leitfaden zur Waldmesslehre. J. D. Sauerländer's Verlag. 266 p. 
Krenn, K. 1948. Tarife zur Massenberechnung von Beständen. Schriftenreihe der Badischen Forstlichen Versuchsanstalt. 6: 1-18.

Laar, A. y A. Akca. 1997. Forest Mensuration. Cuvillier Verlag. Göttingen. 349 p.

Laasasenho, J. 1982. Taper curve and volume functions for pine. spruce and birch. Pub. of Finnish For. Res. Inst. Helsinski. $74 \mathrm{p}$.

Martin, J. 1984. Testing volume equation accuracy with water displacement techniques. For. Sci. 30 (1): 41-50.

Martínez, M. 1948. Los pinos de México. Ed. Botas. 361 p.

McTague, J. 1992. Enhanced estimates of total volume with any single upperstem measurement. For. Eco. Manag. 48: 55-67.
Perry, J. 1991. The pines of Mexico and Central America. Timber Press. 231 p.

Philip, M. 1995. Measuring Trees and Forests. Aberdeen University Press. $338 \mathrm{p}$.

Prodan, M., P. Peters, F. Cox y P. Real. 1997. Mensura Forestal. IICA, BMZ y GTZ. 586 p.

Reed, D. y J. Byrne. J. 1985. A simple variable form volume estimation system. For. Chron. 61: 87-90.

Sloboda, B. 1984. Grundlagen zur Biomathematik und forstlichen Biometrie. Göttingen. 178 p.

Sosa, V. 1976. Tablas de volumen para rodales para la estimación del volumen directamente en el campo. Escuela Nacional de Agronomía. México. 44p.

Turnbull. K., et al. 1963. Comprehensive tree volume tariff tables. Dept. Nat. Res. Olympia. WA. 23 p. 